\title{
Improved Meshing Technique for the Engine Exhaust of an Over-the-Wing Engine and Circulation Control Wing Configuration
}

\author{
John Pham* and David D. Marshalli \\ California Polytechnic State University, San Luis Obispo, CA, 93407-0352
}

\begin{abstract}
This paper details the results of ongoing efforts to improve upon the meshing techniques required to produce accurate RANS CFD solutions for attached and separated flows for a Circulation Control aircraft. Work, thus far, under the current NASA Research Announcement (NRA) project has revolved around an unstructured near-body volume mesh due to its robustness for complicated geometries. However, it has been found that this technique does a poor job capturing detailed flow features such as the boundary layer, shear layer, and wake of large velocity-gradient regions. Its hindrance is primarily due to the limitations of current computational resources, thus new techniques are investigated to improve the quality of CED solutions while not impeding on resources. High quality hybrid near-body volume meshes that combine structured and unstructured meshing have been utilized to meet the goals of the project. The area around the engine and circulation control slots serves as the basis for improved meshing techniques. So far, a hybrid mesh has been successfully generated around the engine and the results of the CF'D aolutions have improved immensely.

The focus of this paper is to show a comparison of the quality of the CFD solution of old and new meshing techniques. In addition, preliminary results of a hybrid mesh around the circulation control slots are discussed and will be the focus of future work. It has been determined that the primary meshing software used, ICEM CFD does not allow enough user control to adaquately reflne particular regions in the flow field, thus, alternative meshing software will have to be explored. Current computing resources limit the total size of the mesh to about 35 million. However, given this constraint, the results clearly show that the hybrid mesh attains more refined and stable CFD solutions.
\end{abstract}

\section{Nomenclature}

$\begin{array}{ll}A & =\text { Area } \\ b & =\text { Wing span } \\ C_{\mu} & =\text { Jet momentum blowing coefficient } \\ h & =\text { Jet slot height } \\ M & =\text { Mach Number } \\ \dot{m} & =\text { Mass flow rate } \\ p & =\text { Static pressure } \\ q & =\text { Dynamic pressure } \\ R & =\text { Gas constant } \\ S & =\text { Wing planform area } \\ t & =\text { Static temperature } \\ U & =\text { Velocity magnitude } \\ y^{+} & =\text {Dimensionless wall distance }\end{array}$

\section{Subscripts}

"Graduate Student, Aerospace Engineering Department, Student Member AIAA

Associate Professor, Aerospace Engineering Department, Senior Member AIAA 


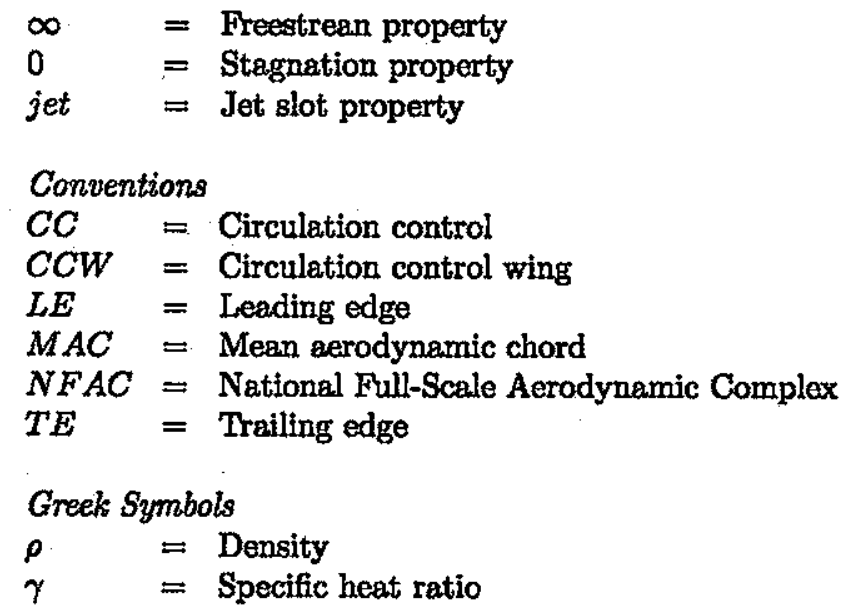

\section{Introduction}

$T^{H E}$ basis for computational analysis in this paper revolves around the implementation and interaction 1 between circulation control wings (CCW) and upper surface blowing (USB). This complex coupling has been studied for years as a way to improve the Short Take-Off and Landing (STOL) performance of an aircraft ${ }^{1}$. STOL configurations are one way researchers are trying to tackle the problem of global air traffic congestion. The problem doesn't appear to be slowing down either as the Bureau of Transportation Statistics recently reported that passenger traffic on U.S airlines increased $1.8 \%$ in August 2010 compared to the same time in 2009. Air traffic congestion causes unnecessary and excess fuel usage in addition to the air and noise pollution associated with it near airports.

\section{A. Advanced Model for Extreme Lift and Improved Aeroacoustics}

The Advanced Model for Extreme Lift and Improved Aeroacoustics (AMELIA) as seen in Fig. 1 is the focus of research at Cal Poly funded under NASA's Subsonic Fixed Wing Program. The collaborative effort culminates into The Integrated Modeling and Verification of Hybrid Wing-Body Low Noise ESTOL Aircraft. Aerodynamic and aeroacoustic analysis will be carried out in an effort to develop and validate predictive modeling capabilities for AMELIA. In addition, a large scale wind tunnel test will be conducted in the 40 foot $\times 80$ foot wind tunnel at the National Full-Scale Aerodynamic Complex (NFAC) at Moffett Field, CA in the fall of 2011. Much of the research thus far for AMELIA revolves around aerodynamics, turbulence modeling, and developing CFD techniques required to reasonably predict accurate CFD solutions. Marshall ${ }^{2}$ gives a complete description of current and future goals of the AMELIA project. Table 1 lists many features AMELIA possess which complicates the meshing process significantly. 


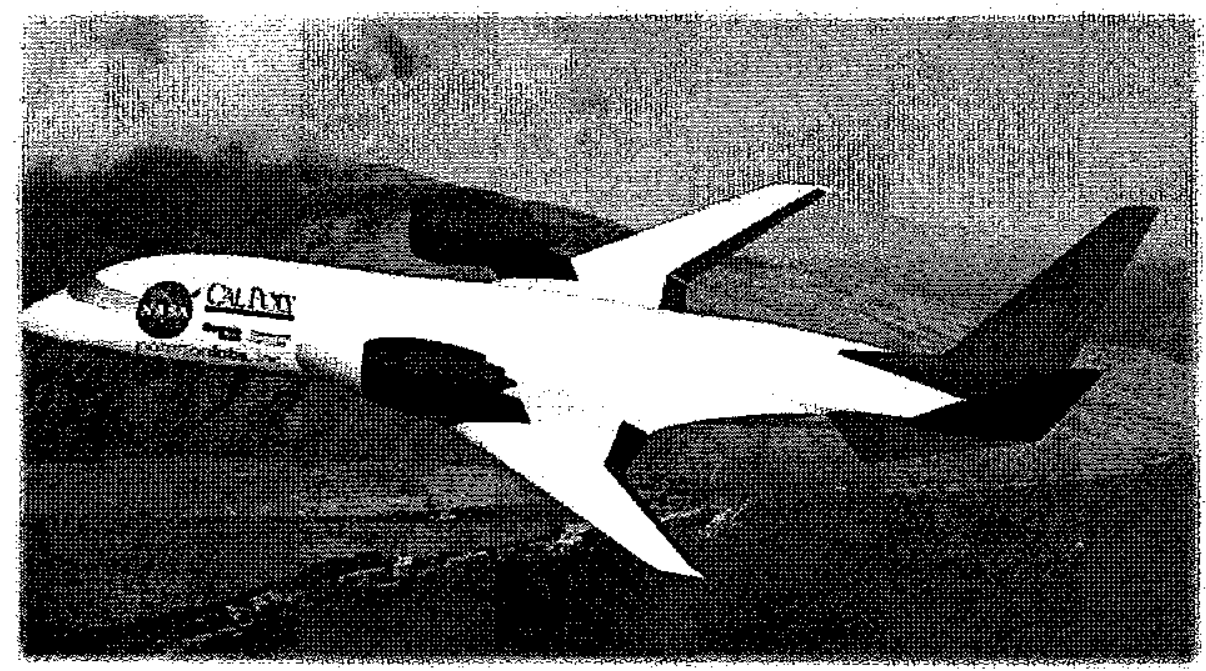

Figure 1. Cal Poly's CESTOL aircraft AMELIA

Table 1. Advanced Features of AMELIA

\begin{tabular}{|l|}
\hline 1. Over-the-Wing Engines \\
\hline 2. Swept Wing \\
\hline 3. Tapered Wing \\
\hline 4. Leading Edge Circulation Control Slots \\
\hline 5. Trailing Edge Circulation Control Slots \\
\hline 6. Deflected Flaps \\
\hline 7. Wind Tunnel Wail Effects \\
\hline
\end{tabular}

\section{B. Circulation Control Overview}

AMELIA utilizes circulation control to generate its STOL capabilities. Lift is traditionally generated for subsonic airfoils through increasing angle of attack and/or camber. However, the increase in lift eventually builds an adverse pressure gradient over the surface of a traditional wing causing the flow to separate and limit the wing's maximum lift coefficient. To overcome this, the use of complex devices such as flaps and slats are utilized at the expense of overall mechanical complexity, noise, and cost.

As a balance, AMELIA implements a circulation control wing (CCW), an active flow control device as a simpler and more effective performance alternative to the usual high-lift devices seen on conventional aircraits. ${ }^{3}$ Figure 2 gives a general overview of the technology while Figure 3 shows the cross section of the CCW with the trailing edge flaps undeflected. The mechanical simplification of circulation control devices over traditional flaps and slats help reduce noise and drag upon takeoff and landing. This is due to large complex wing components no longer obstructing the freestream flow. The largest effect of using CCW will be seen during takeoff and landing where high lift coefficients are needed at low airspeeds. This technology is made possible through the use of the Coanda effect along the adjacent curved surface of the wing, where the accelerated fluid is able to stay attached for an extended amount of time, thus delaying separation and increasing lift. ${ }^{4}$ Moreover, the camber of the airfoil and dual radius flap are manipulated such that the stagnation point on the leading edge and flow separation point on the trailing edge provides a positive circulation around the wing and consequently increasing lift. In addition, initial research into the flow physics of AMELIA reveals additional unexpected lift through the engine exhaust entrainment due to the downward momentum strength of the circulation control jet stream. This can be seen in Figure 4 and comes from the work of Blessing. ${ }^{5}$ Overall, AMELIA produces lift through three sources: 1). Traditional lift through airfoil production 2). Circulation control slots through Coanda effect 3). Engine exhaust entrainment similar 
to Upper Surface Blowing technology. The increased lift production observed can improve immensely the takeoff and landing performance of aircrafts and can enable large aircrafts to operate out of smaller airports, thus alleviating the growing problem of air traffic congestion. The extent of the stagnation and separation point movement is primarily a function of the jet momentum coefficient, $\mathrm{C} \mu$, which is a measure of the jet momentum relative to the freestream momentum. It is represented by Eqn. (1).

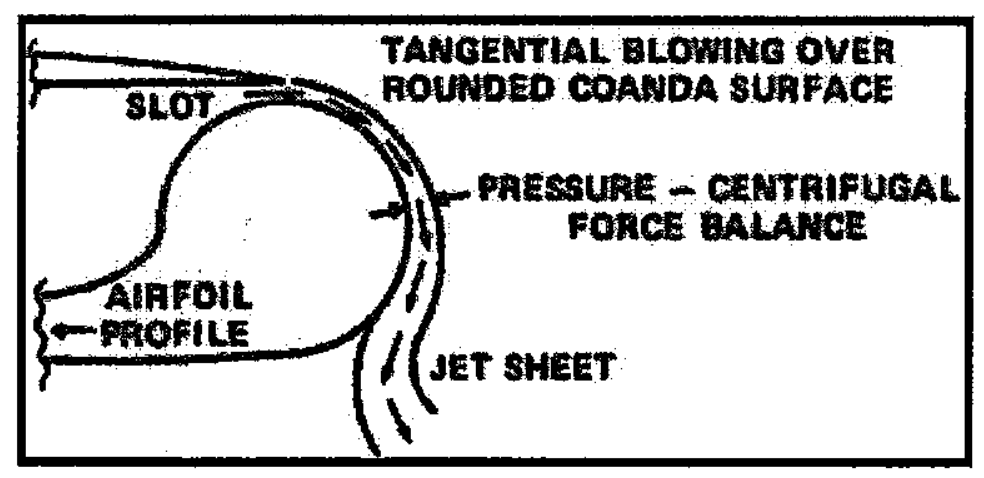

Figure 2. General characteristics of circulation control technology

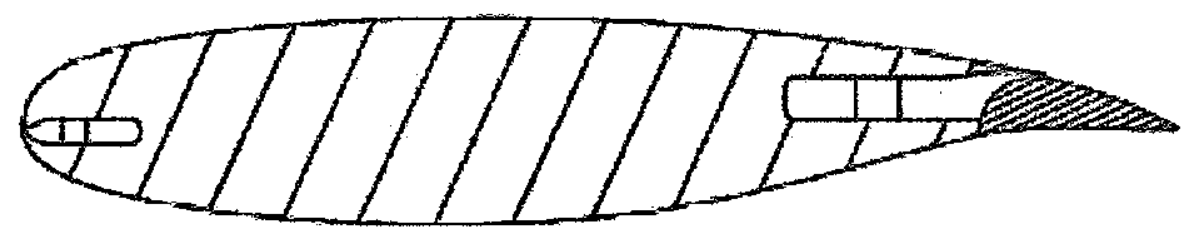

Figure 3. Circulation control airfoll with leading edge and tralling edge slots

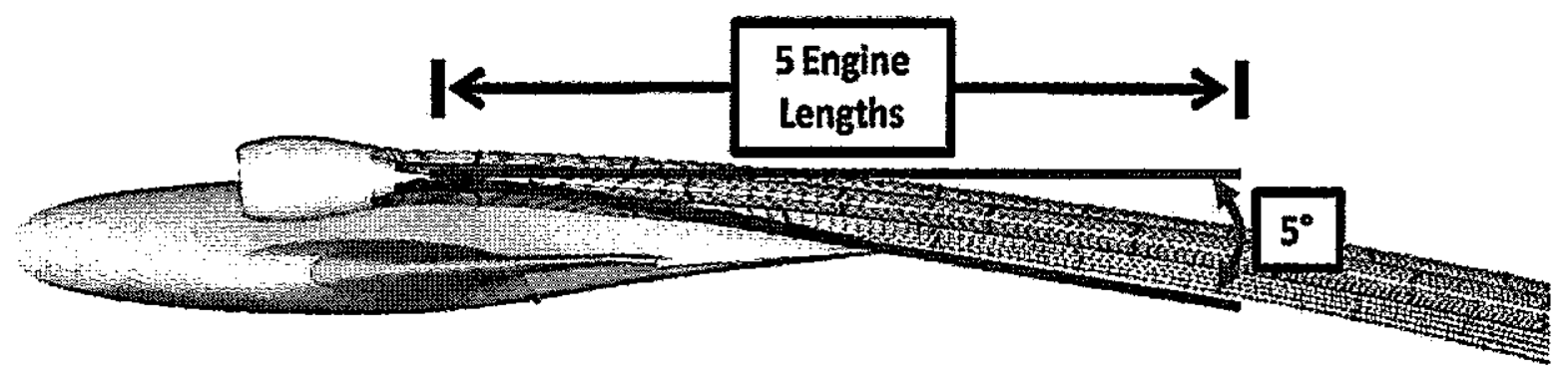

Figure 4. Engine exhaust entrainment due to downward momentum strength of circulation control jet stream

\section{Model Description}

The following section will discuss the model used during experimental testing and for computational analysis in this peper.

\section{A. Experimental Model}

The experimental aspect of this contract will culminate in a full scale wind tunnel test of the AMELIA model. An in depth description of the experimental model as well as overall project details is provided by Marshall et al. ${ }^{2}$ Figure 5 shows a cut away view of the wind tunnel model, highlighting the leading and trailing edge circulation control slots as well as the TPS unit. 


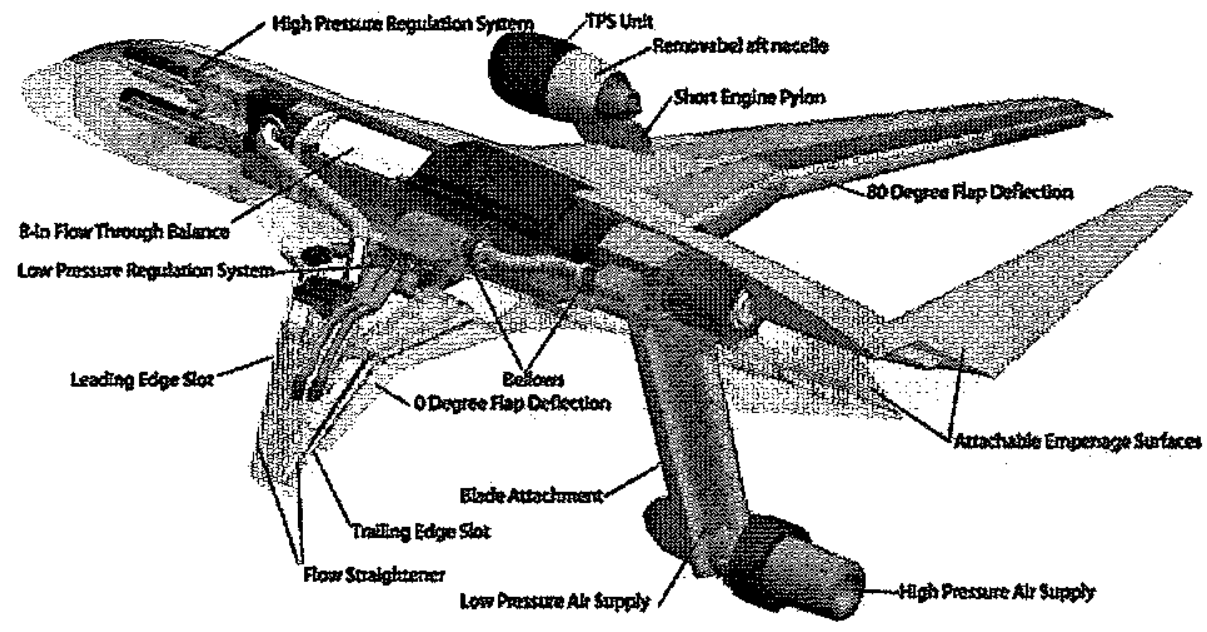

Figure B. Schematic of AMELIA experimentel model

\section{B. Computational Model}

For the computational analysis, many features from the experimental model were removed to simplify and obtein reasonable meshes. Figure 6 shows the simplified computational model of AMELIA. Features that were removed include the wind tunnel sting, engine pylon, and fuselage blend. All of which were deemed negligible on the aerodynamic effects of AMELIA. Even after simplification of the model, AMELIA possesses many features that pose a challenge on the meshing process.

The first is the "scissor-like junction" seen in Fig. 7 that the flaps make with the wing when deflected for takeoff and landing configurations. Generating an error-free high quality boundary layer mesh near these two junctions has become very difficult. The most complex feature of AMELIA is the circulation control slots located on the leading edge and trailing edge of the wing seen in Fig. 8. The slot varies in height from inboard to outboard of the wing, where the smallest is 0.137 inches. The mean aerodynamic chord (MAC) of AMELIA is 18.6 inches, which makes the smallest gap in the slot $0.74 \%$ of the MAC. The drastic change in size from the slots to the remaining wing makes it very challenging to generate a good mesh that is within the limitations of existing computational resources. The last troubling issue about AMELIA is the large change in flow speed relative to freestream in the wake of the trailing edge circulation control slots and the engine. The ratio of flow speed of the slot wake to freestream flow is about 6 , while the engine wake's ratio to freestream flow is about 4. These large flow speed ratios generate a strong shear layer that's been measured from the preliminary CFD solutions to travel at least 20 chord lengths downstream. The challenge this poses is that the mesh in the region of the shear layer and wake must be fine enough to capture the details of the flow feature, but existing computational resources limit the number of mesh elements that can exist in the computational model. 


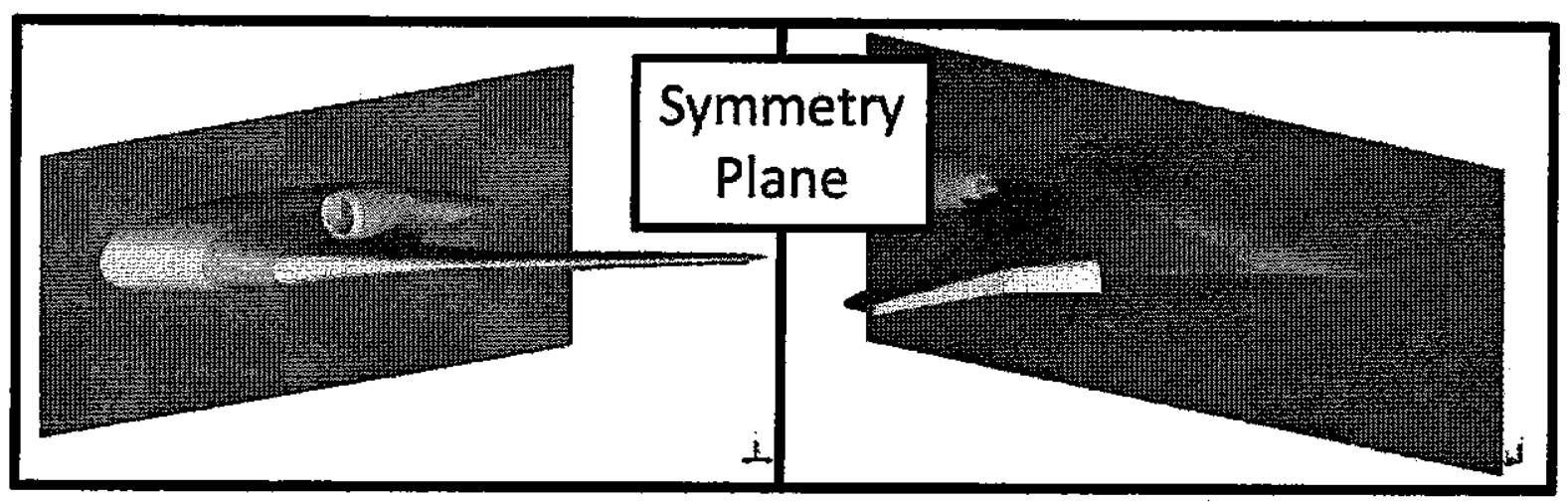

Figure 6. Simplifled computational model of AMELIA

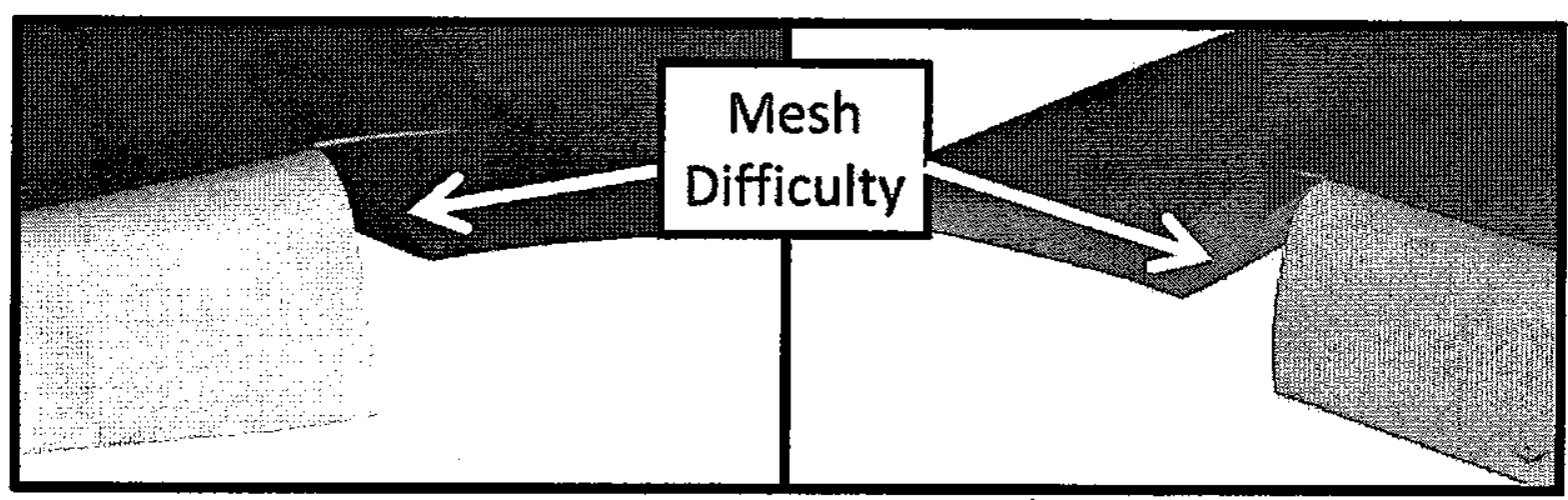

Figure 7. Difflcult "sclssor-junction" - outboard (left) Inboard (right)

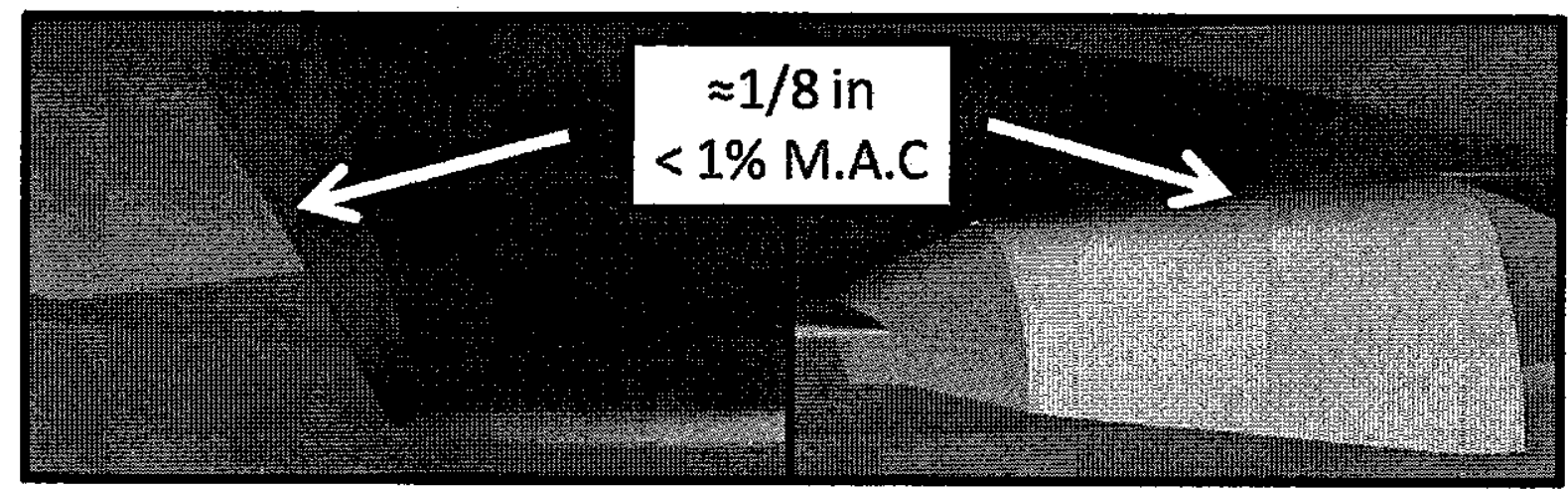

Figure 8. Circulation control slots - leading edge (left) traillng edge (right)

\section{Mesh Generation}

The mesh generation software chosen for this NRA is ICEM CFD. ${ }^{6}$ Careful mesh generation must be taken to ensure that the final mesh exhibits good quality elements. The quality of the mesh has significant implications on the convergence, stability, and accuracy of the numerical simulation. The type of mesh chosen can also determine the success or failure in attaining a computational solution. The mesh must be sufficiently fine to provide an adequate resolution of the important flow features and geometry topology. Recirculation vortices and steep flow gradients within the viscous boundary layer have been observed in preliminary CFD 
solutions, thus requiring proper mesh resolution where these flow features are expected. The final mesh used for numerical simulation consists of four key components. The first is a high-quality surface mesh that maps itself to the computational model followed by a boundary layer and shear layer mesh that can capture complex viscous effects. Then there needs to be a refined near-body volume mesh that can capture the unsteadiness of the flow passing over the computational model. Lastly, there needs to be a far-body volume mesh resolved enough to capture flow features expected to extend many chord lengths downstream of the computational model such as wingtip vortices, circulation control flow, and engine exhaust. In addition, the far-body volume mesh must be large enough to allow these secondary flow features to dissipate into the freestream flow.

Since the beginning of the NRA project, the meshing approach has revolved around a near-body unstructured volume mesh. This approach (1st Method) hes been utilized for its robustness of modeling complex geometries like AMELIA and has yielded encouraging results of the general nature of the circulation control wing interacting with the engine. However, this approach has done a poor job capturing important flow features near the computational model that are essential to producing the most accurate validation data for the future wind tunnel test. The mesh regions that need to be improved first are in areas of large flow gradients such as the engine exhaust and high speed air accelerated through the circulation control slots. Work under this NRA project that uses the 1st Method can be seen in Blessing et al., ${ }^{5,7}$ Marcos et al. ${ }^{8,9}$ and Lichtwardt et al. ${ }^{10}$ In addition, there has been 2-dimensional work that has utilizes fully structured meshes and can be seen in Lane et al., ${ }^{11}$ Golden et al. ${ }^{12}$ and Storm et al. ${ }^{13}$ The work from this paper has built and improved upon the methods learned thus far in simulating circulation control flowfileds.

For the latest approach (2nd Method), it has been proposed to generate a structured mesh in certain regions to improve the mesh quality. The structured regions will be merged with unstructured elements to make a hybrid mesh. The 2nd Method provides much more user-control over meshing parameters which become important in capturing complex flow regions, but it comes at the expense of time. Although it takes significantly longer in creating a structured mesh than an unstructured mesh, it is a necessity in obtaining the most accurate solution possible and offers many benefits over the 1st Method. The areas of most concern are the region around and behind the following: (1) engine (2) trailing edge circulation control slots (3) leading edge circulation control slots. This peper focuses on improvements made to the mesh around the engine.

The following sections will discuss the mesh generation procedure highlighting the four key components mentioned previously. The first two sections discuss the near-body mesh generation of the 1st and 2nd Method. The last section will discuss the far-body mesh generation that has been used for both methods. The total mesh count of the 1st Method was about 34 million elements, while the 2nd Method was about 36 million elements.

\section{A. Near-Body Unstructured Meshing (1st Method)}

The 1st Method uses the Octre Algorithm within ICEM CFD to generate the surface and near-body volume mesh. This approach begins with a tetrahedron that encloses the entire computational model and is subdivided until all mesh size requirements set on the surface and volume of the model have been met. This top-down approach allow for faster mesh generation because it only refines the mesh where necessary, while maintaining larger elements everywhere else. The Octree method is great for computing a preliminary CFD solution for understanding the general flow features of the computational model. However, when generating validation data for a wind tunnel test, all complex flow features of the model must be captured and that's where the Octree method does a poor job. This has been improved upon and will be discussed in a later section of this paper.

\section{Surface Mesh}

There are various ways to generating a surface mesh. One can use all structured elements, all unstructured elements, or a combination of both. Unstructured surface meshing was chosen as it is the easiest and fastest. There are two methods in ICEMCFD for creating this surface mesh: 1) patch-dependent 2) patchindependent. The patch-dependent method requires a high-quality CAD model and tedious mesh size curve setting in order to generate a proper mesh. With more time, this method would be used because it yields a higher-quality surface mesh and allow the user more control over mesh parameters. The patch-independent method is best for low quality CAD models with poor connectivity. The surface mesh is created using the 
top-down Octree method which requires minimal input from the user and thus can be created very quickly. To start the Octree method, mesh sizes must be set for each part in the computational model. Parts that do not present severe curvature, such as the fuselage, flat surfaces of the wing and the tail, were set with coarse sizes. Surfaces that do present a lot of curvature, such as the leading edge and trailing edge of lifting surfaces are meshed with finer sizes so that the topology of the geometry can be captured.

The circulation control slots exhibited by the leading and trailing edge of the wing are the most novel features of this model. The air accelerated from these slots produce a strong viscous dominated region downstream. It is essential that these slots are adequately resolved in order to capture a high resolution flow field. Figure 9 shows the surface meshes of the forward and aft end of the wing using the Octree method.

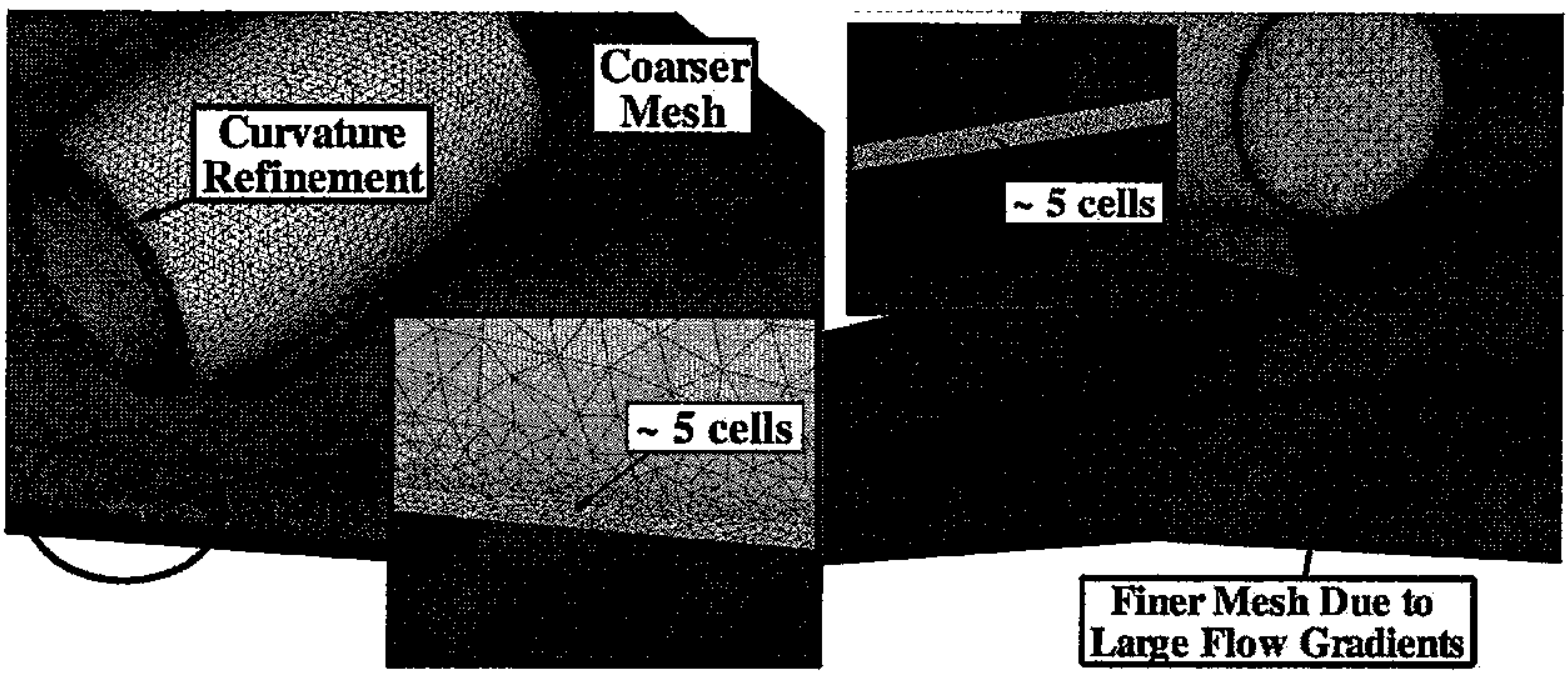

Figure 9. Unstructured surface mesh of wing, cfrculation control slots, \& engine

\section{Boundary Layer Mesh}

To create the near-wall mesh, prisms are generated off the surface triangles. Prisms are used instead of tetrahedrals because they can be easily adjusted in accordance with the near-wall turbulence model requirements which will become an important issue in accurately capturing viscous effects. For most applications, it is acceptable to model the boundary to have at least one element within the fully turbulent log-law layer and allow the solver to use wall functions ${ }^{14}$ to bridge the gap between the surface of the model and the fully turbulent log-law layer. Wall functions will be discussed further in Section V. This method will be taken in order to obtain solutions more rapidly. As the mesh and solver settings become more evolved, modeling the mesh near the wall will migrate from the wall functions approach to a much more refined mesh near the wall so that the viscous dominated region can be fully resolved.

The high quality mesh that is desired will require careful consideration to cell shape in terms of aspect ratio, skewness, and warp angle. If care is not taken in the mesh setup, prism elements will exhibit very poor quality in these categories. Elements with large aspect ratios should always be avoided in critical regions of the flow field. These types of elements can degrade the solution accuracy and may result in instability of the simulation. As for skewed elements, this will be inevitable. It is up to the user to maintain as little as possible the number of elements below a certain skewness quality. For complicated geometries like AMELIA that contain very small features, this has been very difficult. Most mesh-generating packages have built-in smoothing algorithms that can help remedy the complications expected to be seen in building prism elements on the model.

There will be prisms, tetrahedral, and hexahedral elements used to model the volume of the computational domain. To ensure a good mesh, the user needs to consider volume transition ratio. For a good volume transition from the surface triangles to the volume elements, each prism element follows a $40 \%$ volume growth ratio. As for the interface between the prisms and tetrahedrals, the user should aim to have the last prism element be roughly 1:1 in volume ratio to the adjacent tetrahedral element. Figure 10 shows an example of how prism elements are to be mapped for each surface of the aircraft model. 


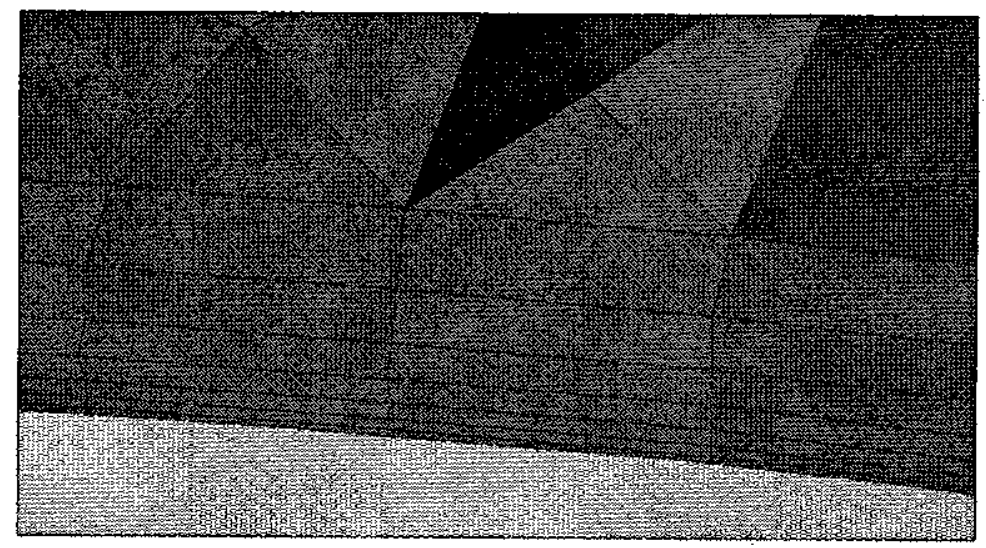

Figure 10. Prism boundary layer mesh bridging surface trlangles and volume tetrahedrals

\section{Volume Mesh}

Constructing a good mesh in the region near the computational model will be important as it presents a lot of the unsteadiness that is expected to occur in the numerical simulation. Thus, the user must be extra careful choosing the proper techniques to model this region. Typically, there are three methods: (1) fully unstructured (2) fully structured (3) hybrid. This section outlines the use of fully unstructured meshing while Section B will discuss the hybrid method.

Tetrahedral elements are well suited for handling arbitrary shape geometries. This proves to be a major plus as the AMELIA configuration contains complex features. Employing unstructured tetrahedral meshing to the domain will inherently increase the total cell count because it has be to adequately fine enough to resolve the important flow features in that region. As mentioned previously, the near-body volume mesh is created using the top-down Octree Algorithm. Figure 11 shows a full cutplane view of the near-body volume mesh. These elements extend up to 2 chord-lengths in all directions away from the nearest point on the computational model to anticipate the highly unsteady flow regime that surrounds the model.

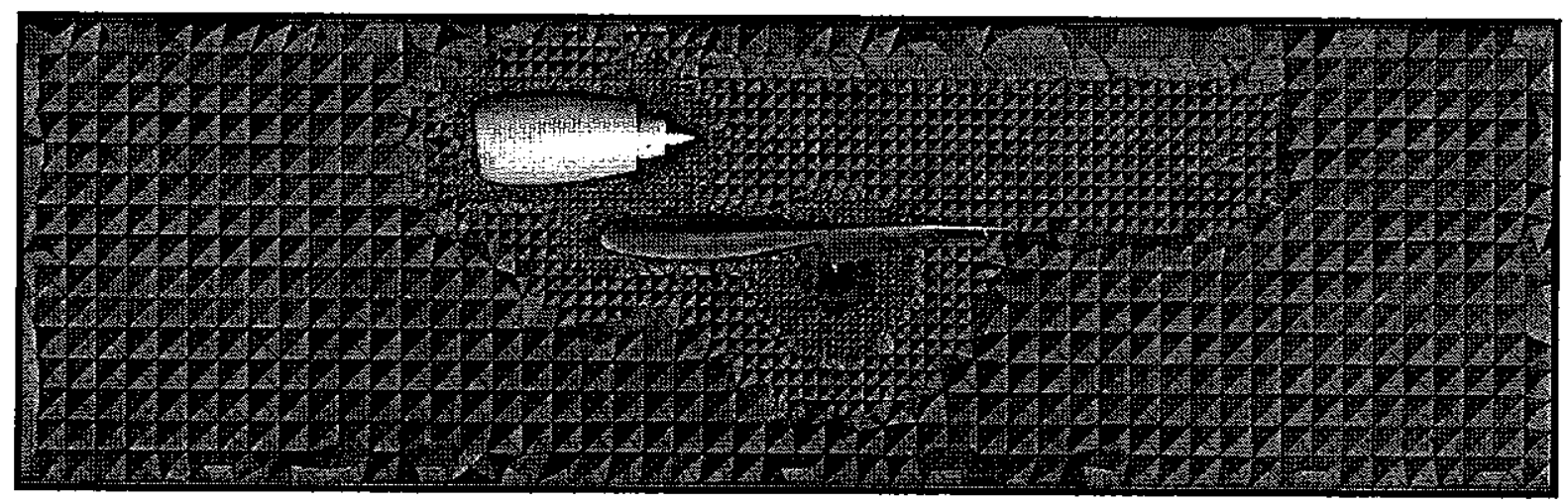

Figure 11. Cutplane of near-body unstructured volume mesh

\section{B. Near-Body Hybrid Meshing (2nd Method)}

This section outlines the improvements to the meshing techniques previously discussed. For the first improvement, the algorithm chosen to generate the unstructured tetrahedral elements has been improved. The 2nd Method now uses the TGrid Delaunay Algorithm6 instead of the Octree Algorithm. Unlike the Octree method, the Delaunay method creates a tetrahedral mesh using a bottom-up approach from an existing surface mesh. This approach creates a smoother transition in the volume element size. The second improvement is the creation and merging of a structured mesh around the engine. This type of mesh creates quadrilaterals on the surface of the model and hexahedrals in the volume. The Hybrid meshing approach 
improved the quality of the mesh around the engine immensely, while staying within the limitations of computational resources.

\section{Surface Mesh}

As mentioned, a surface mesh must exist in order to run the Delaunay method. The surface mesh was created using the Octree method and then deleting the volume elements afterward. To create the structured mesh around the engine, it must be prepared with a structured surface mesh. A multi-block scheme was created around and behind the engine where mesh sizes were manually set to match user requirements. The blocking scheme can be seen in Fig. 12. The surface mesh of the engine can be seen in Fig. 13 compared to the unstructured surface mesh used in the 1st Method. The structured approach improved the quality of the mesh, while also reducing the cell count by $30 \%$.

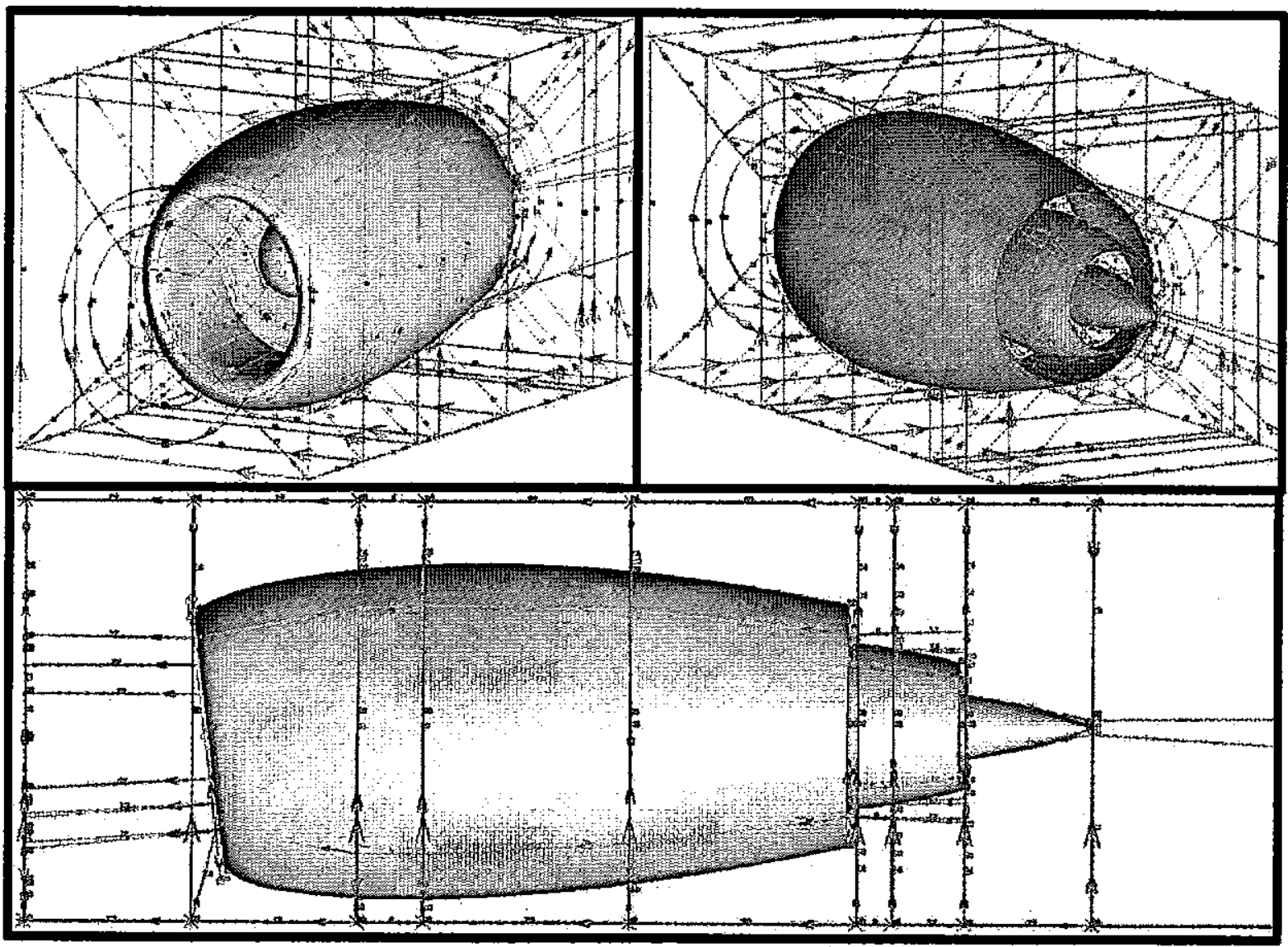

Figure 12. Multi-blocking around and behind the engine 


\section{9,000 elements}

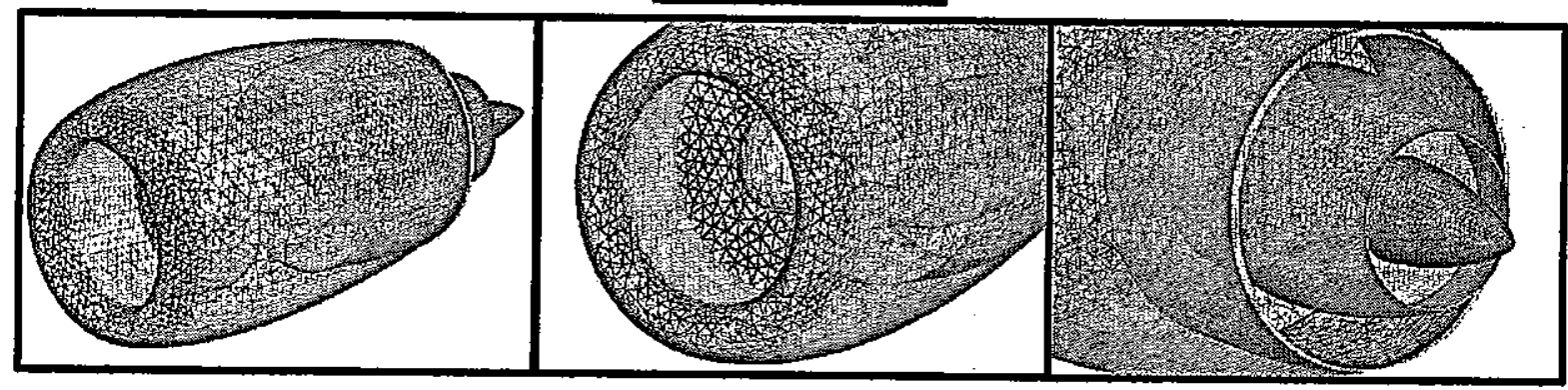

\section{1,000 elements}

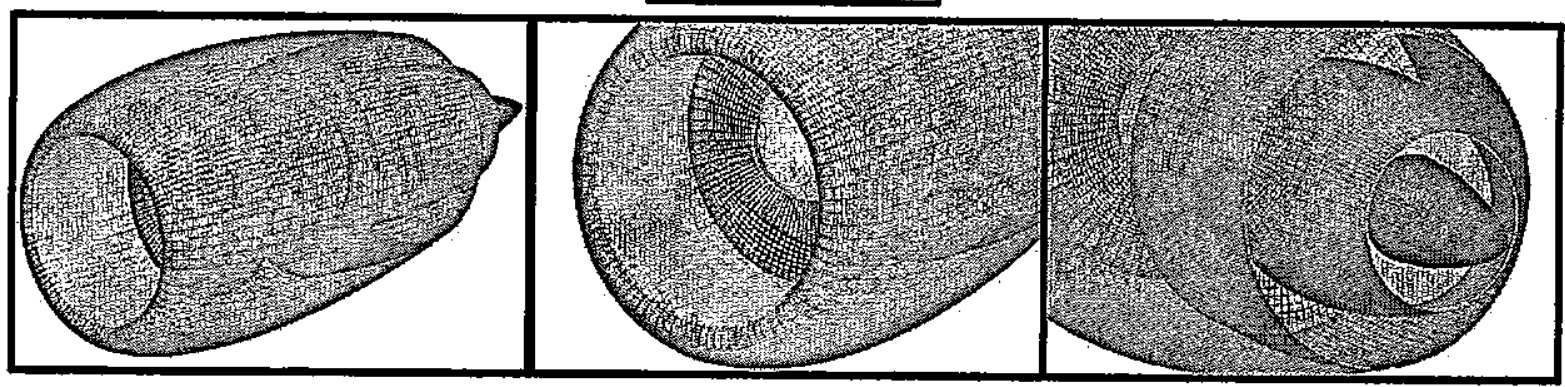

Figure 13. Surface mesh comparison of enginet unstructured (top row) structured (bottom row)

\section{Boundary Layer Mesh}

Numerical simulation of high Reynolds number flow creates strong gradients normal to the surface of the computational model and across shear layers. Modeling the boundary layer and shear layer adequately is one of the most crucial aspects of a high-quality mesh. To model these regions correctly, the mesh resolution should be much finer compared to the average resolution of the model. In order to keep the mesh size manageable, anisotropic elements are needed, thus, the biggest benefit to creating a structured mesh is the amount of control the user has over mesh parameters. This allows the user to control how much refinement is needed in all directions relative to the surface of the model. A comparison of the boundary layer and shear layer mesh around the engine can be seen in Fig. 14. Creating a boundary layer mesh using the 1st Method is decent. Users can control the height, number of layers, and growth ratio as you can with a structured mesh. However, one thing the user cannot control is the transition of prism layers from one section to another of the computational model. When it comes to modeling the shear layer, the 1st Method does a very poor job because the user cannot directly control the mesh density of a particular region like the 2nd Method. However, ICEM CFD does heve a feature that allows the user to create "density regions" where the user can set a mesh size limit. Doing this would have increased the total mesh count significantly, which would require a compromise in other areas of the mesh in order to stay with computational limitations. 


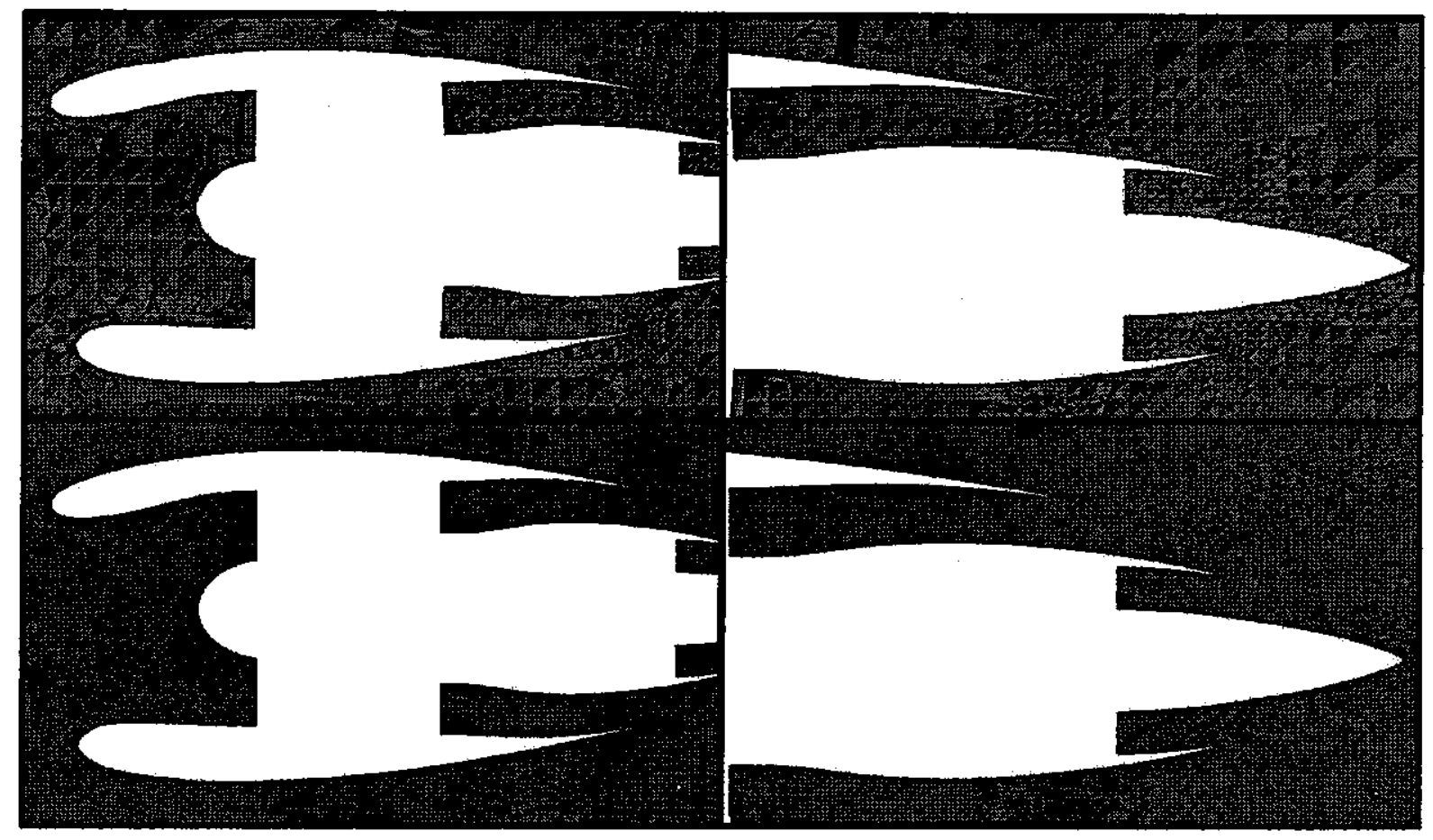

Flgure 14. Boundary layer mesh comparison of engine - 1st Method (top row) 2nd Method (bottom row)

\section{Volume Mesh}

There are two benefits of the 2nd Method that stand out most. First, the quality of the mesh with respect to aspect ratio, skewness, and orthogonality are drastically improved with increased user-control in mapping the mesh to the geometry. And secondly, the surface mesh count of the engine was reduced by about $30 \%$. This reduction allows for further mesh refinement around other important flow features of the geometry without impeding on limited computational resources. A full cutplane of the near-body hybrid volume mesh can be seen in Fig. 15. It should be noted that the structured and unstructured portions of the mesh are created separately and merged together. When merging two separate mesh topologies, it becomes important that there is a good transition from one mesh to the other. This was not addressed for this paper, but will be improved upon in the near future. Figure 16 compares the near-body volume mesh of the 1st and 2nd Method, where the most prominent improvement being the resolution of the boundary layer, shear layer, and wake mesh of the engine.

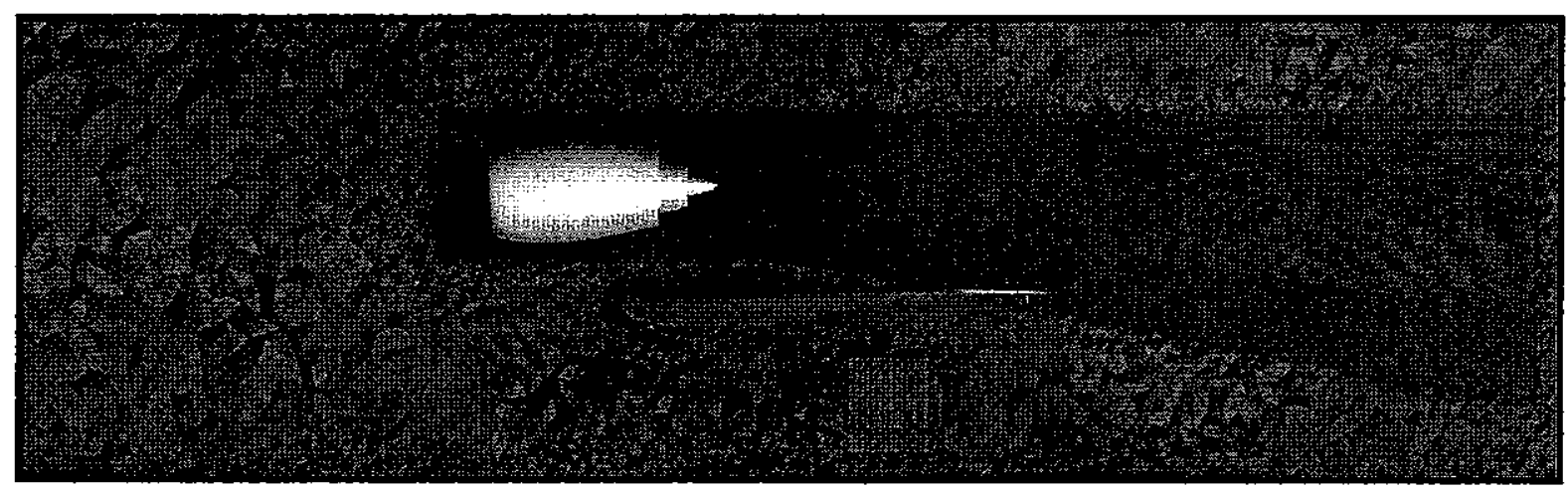

Figure 15. Cutplane of near-body hybrid volume mesh 


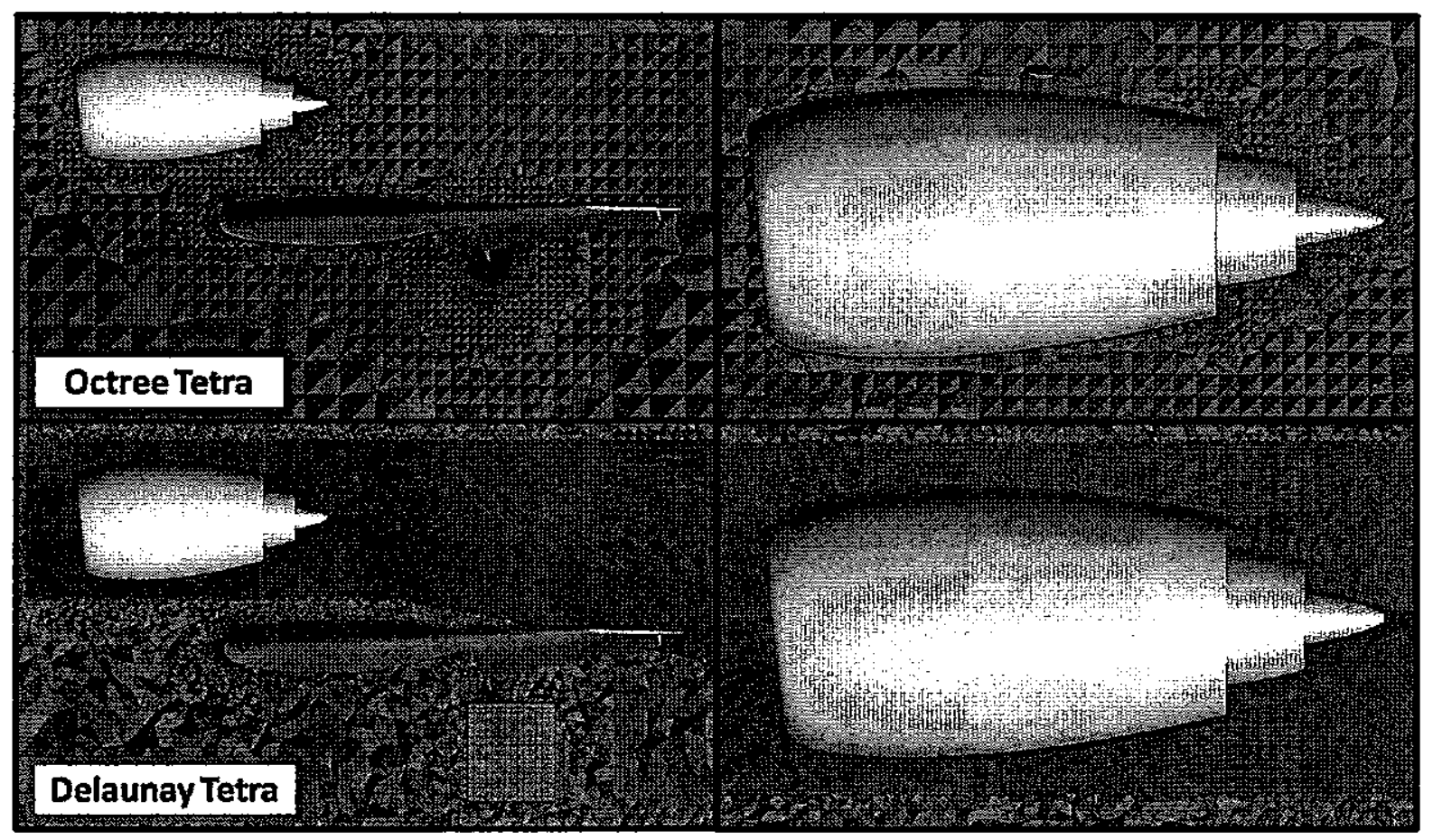

Figure 16. Volume mesh comparison of engine wake - 18t Method (top row) 2nd Method (bottom)

\section{Far-Body Structured Meshing}

To model the flow field that is away from the unsteady flow regime, hexahedral elements are the most efficient way to capture the secondary flow features. Also, this region will not need to map to the surface of computational model, so this method can be generated very easily. Both near-body meshing approaches utilized the same far-body structured mesh.

The mesh was assembled using a number of structured blocks attached to each other. Using this multiblocking approach gives the user a lot of flexibility in choosing how to map out each structured block. Perhaps the user wants to refine a certain block but does not need the same mesh resolution in a different block. For example, it is expected that the flow field will exhibit secondary flow features such as wingtip vortices and high momentum slot exhaust. These vortices are known to travel many chord lengths downstream of the model before dissipating. In subsonic flow, failure to capture this feature accurately may greatly affect the final computational solution. Thus, it would be advised that the blocks that represent the wake of the model be much more resolved. Figure 17 shows an example of how the blocking scheme looks like.

Instead of using hexahedral elements, the user could use unstructured tetrahedral elements to model this portion of the domain. However, doing so would diminish the accuracy of the solution and would unnecessarily increase total cell count. Using preliminary CFD solutions as guidance, the structured domain was modeled to be roughly 45 chord-lengths upstream and 65 chord-lengths downstream. Upward and downward is modeled to be 20 feet from the model to represent the wind tunnel test section of the NFAC. An example of the hybrid mesh used is shown in Fig. 18. 


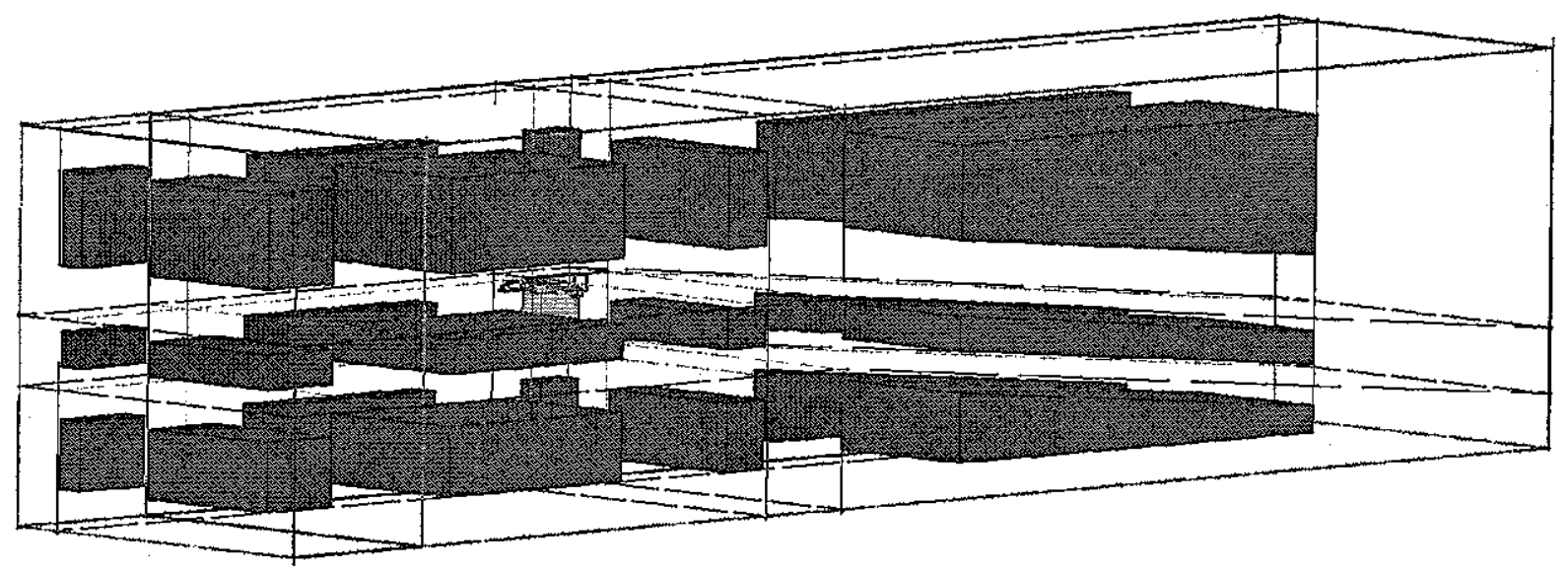

Flgure 17. Multi-block scheme for structured meshing

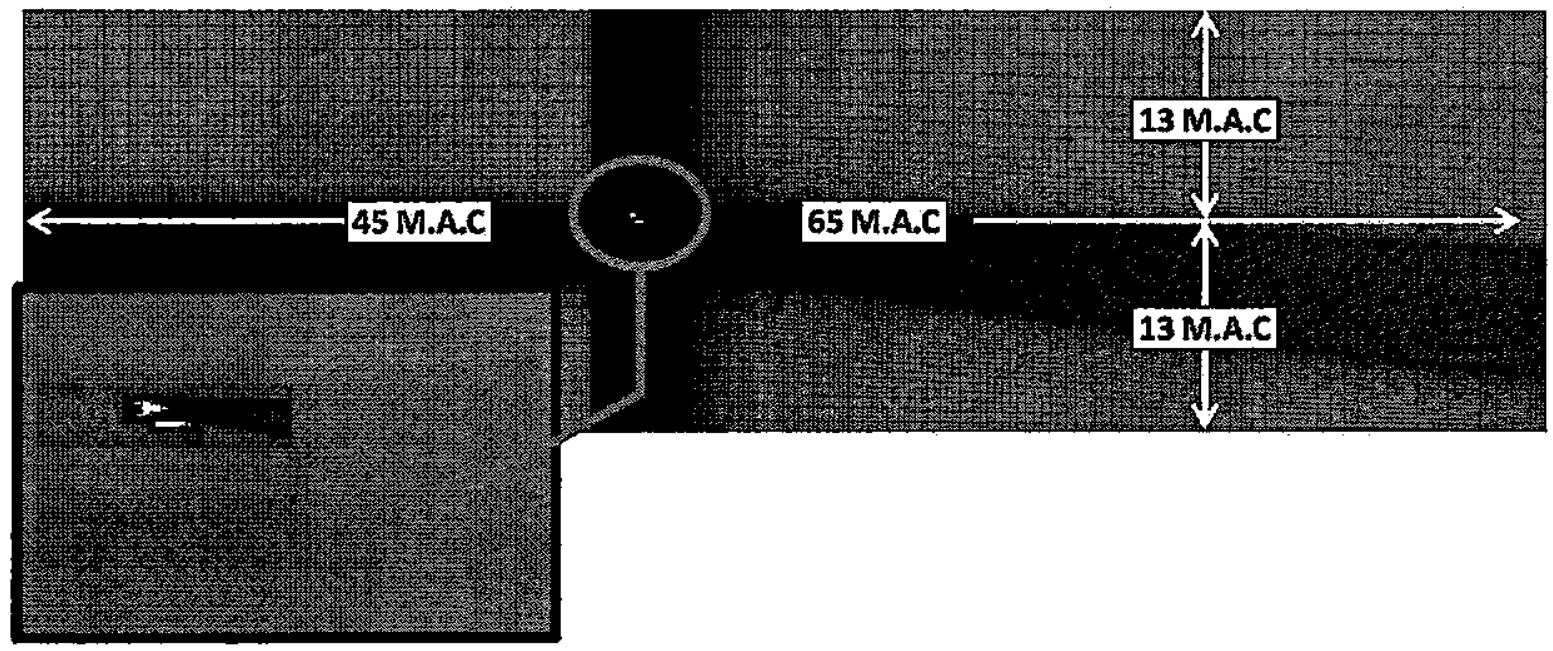

Flgure 18. Cutplane of structured far-body volume mesh

\section{Numerical Simulation Setup}

The following section outlines the solver settings and boundary conditions chosen to setup each computational analysis.

\section{A. Solver Settings and Boundary Conditions}

The numerical solver chosen for this project is FLUENT. ${ }^{15}$ The numericel scheme chosen for the 3-D cases is an implicit compressible solver that couples and solves the governing equations of continuity, momentum, and energy simultaneously. The coupled solver is generally used for compressible flows and because the flow exiting from the slots are compressible, this solver proves to be the best choice. In addition, both cases were simulated using an inviscid model and iterated up to 15,000 steps. With more time, the viscous model will be turned on and allow the solution to further progress. Table 2 summarizes the solver settings used for the model. 
Table 2. General FLUENT zolver settings

\begin{tabular}{|l|l|}
\hline Model & Inviscid \\
\hline Solver & Compressible \\
\hline Density calculator & Ideal Gas Law \\
\hline Viscosity calculator & Sutherland's Law \\
\hline Reference length & $4.3 \mathrm{ft.}$ \\
\hline Reference area & $5.8 \mathrm{ft.}^{2}$ \\
\hline
\end{tabular}

The boundary conditions employed on the model also have serious implications on the computational solution and are very difficult to define the correct boundary conditions to mimic the real physical representation of the flow field. Initial and boundary conditions are essential to solving the governing equations, thus, meaningful numerical solutions are highly dependent on the types of boundary conditions implemented and the values that initialize that boundary. For CCW simulations, jet slot boundary conditions must be specified to simulate the jet flow effects. Generally, the driving parameter for CCW simulations is the momentum coefficient, $\mathrm{C} \mu$, defined as the following,

$$
C_{\mu}=\frac{\dot{m} U_{j e t}}{\frac{1}{2} \rho_{\infty} U_{\infty}^{2} S_{r e f}}
$$

Or alternatively as the following,

$$
C_{\mu}=\frac{\rho_{\text {jet }} U_{\text {jet }}^{2} A_{\text {jet }}}{\frac{1}{2} \rho_{\infty} U_{\infty}^{2} S_{\text {ref }}}
$$

In part with this NRA project, Georgia Tech Research Institute (GTRI) conducted studies of a CCW wing and concluded that a $\mathrm{C} \mu$ between $0.4-0.6$ is most optimal. The results of this study is documented in Marshell. ${ }^{16}$ However, there is no boundary condition in FLUENT that allows for the specification of $\mathrm{C} \mu$. The best available option is to specify the slots as a. Pressure-Inlet boundary condition, where FLUENT requires the total pressure and temperature at the slot. The total temperature is assumed to be approximately equal to the total temperature of freestream. Obtaining the total pressure at the slot requires a few calculations. The slot faces are assumed to be the throat of the nozzle, which implies the local Mach number at the jet should be unity. The static temperature of the slot can be computed using the following equation.

$$
\frac{t_{0, j e t}}{t_{j e t}}=1+\frac{\gamma-1}{2} M_{j e t}^{2}
$$

Equation (3) can also be re-written to be the following,

$$
U_{j e t}^{2}=\frac{2 \gamma R}{\gamma-1}\left(t_{0, j e t}-t_{j e t}\right)
$$

From Ideal Gas Law, the following relation can be obtained,

$$
\rho_{\text {jet }}=\frac{p_{\text {jet }}}{R t_{\text {jet }}}
$$

Substituting Eqns. (4) and (5) into Eq. (2), we get the following equation where the jet static pressure can be computed using a desired $\mathrm{C} \mu$ of 0.4 ,

$$
C_{\mu}=\frac{2 \gamma}{\gamma-1} \frac{A_{j e t}}{q_{\infty} S_{r e f}} \frac{p_{j e t}\left(t_{0, j e t}-t_{j e t}\right)}{t_{j e t}}
$$

With static pressure known, the follow relation can be used to compute the total pressure at the slot.

$$
\frac{p_{\text {jet }}}{p_{0, j e t}}=\left(1+\frac{\gamma-1}{2} M_{\text {jet }}^{2}\right)^{-\frac{\gamma}{\gamma-1}}
$$

Table 3 shows boundary conditions used for the engine and slots along with the values used at that boundary. The engine boundary conditions come from Blessing. ${ }^{5}$ From a simple 2-D study of a CC airfoil, 
the values computed from the solution compares very well with the values predicted using 1-D isentropic relations. Figure 19 illustrate the desired boundary conditions for the engine and circulation control slots.

Table 3. Boundary conditions

\begin{tabular}{|l|l|}
\hline \multicolumn{2}{|l|}{ Freestream Conditions } \\
\hline Boundary condition & Pressure-Far-Field \\
\hline Pressure & $14.7 \mathrm{psi}$ \\
\hline Density & $0.0765 \mathrm{lbm} / \mathrm{ft}^{3}$ \\
\hline Mach Number & 0.07 \\
\hline (1) Engine Inlet \\
\hline Boundary condition & Pressure-Outlet \\
\hline Static pressure & $11.5 \mathrm{psi}$ \\
\hline Total temperature & $520.7 \mathrm{R}$ \\
\hline (2) Engine Fan Outlet \\
\hline Boundary condition & Mass-Flow-Inlet \\
\hline Mass flow rate & $4.03 \mathrm{lbm} / \mathrm{s}$ \\
\hline Total temperature & $421.92 \mathrm{R}$ \\
\hline (3) Engine Nozzle Outlet \\
\hline Boundary condition & Mass-Flow-Inlet \\
\hline Mass flow rate & $1.32 \mathrm{lbm} / \mathrm{s}$ \\
\hline Total temperature & $579.33 \mathrm{R}$ \\
\hline (4) Leading Edge Circulation Control Slot \\
\hline Boundary condition & Pressure-Inlet \\
\hline Total pressure & $25.2 \mathrm{psi}$ \\
\hline Total temperature & $519.7 \mathrm{R}$ \\
\hline (4) Trailing Edge Circulation Control Slot \\
\hline Boundary condition & Pressure-Inlet \\
\hline Total pressure & $25.2 \mathrm{psi}$ \\
\hline Total temperature & $519.7 \mathrm{R}$ \\
\hline
\end{tabular}




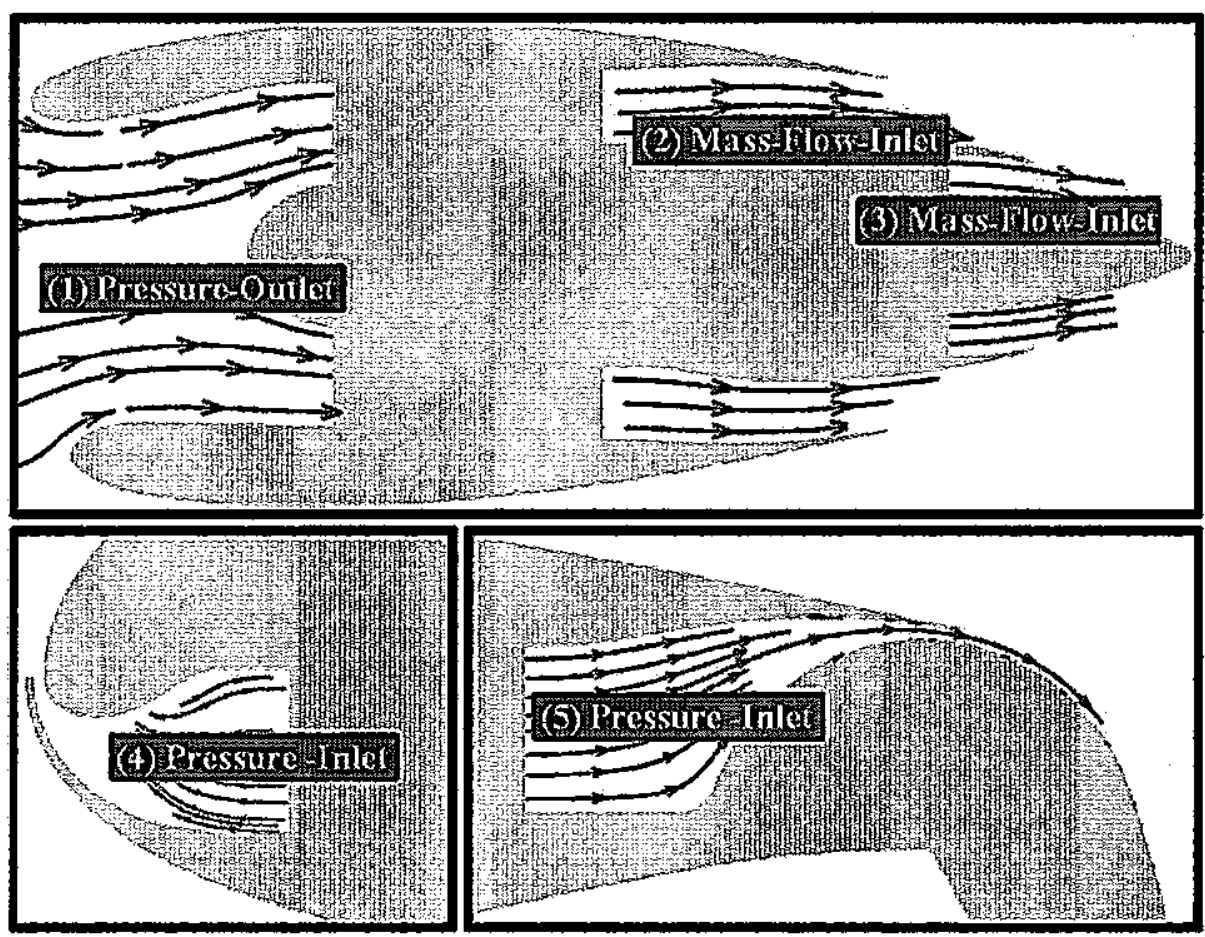

Figure 19. Boundary conditions aet on engine and circulation control slots

\section{Results \& Discussion}

This section will discuss the CFD results of the two different meshing techniques discussed so far in this paper. The next series of figures display the solution of both cases at a cross-sectional plane through the centerline of the engine. In addition, both solutions were stopped at 15,000 iterations to allow for comparison. Figure 20 shows contours of velocity for both cases. Immediately, there are a few problems we see visually with the unstructured mesh. First off, the engine exhaust streams from the fan and nozzle exits are poorly defined and smeared away by the unstructured elements. The mass flow rates of fan and nozzle exits are $1.83 \mathrm{~kg} / \mathrm{s}$ and $0.60 \mathrm{~kg} / \mathrm{s}$, respectively. The 3 -to- 1 ratio should show a discernible difference of the two exhaust streams which the unstructured case doesn't clearly show. The next problem seen is the poor resolution of the engine exhaust wake about 3.5 engine lengths downstream. When compared to the structured case, the solution appears well-defined and is not dissipated by the poor mesh quality of the unstructured mesh. And then lastly, the jet stream of the trailing edge slot appear to be unstable and oscillatory in the unstructured case. Not much effort was spent refining the structured region in the trailing edge wake, however, the solution still exhbits more stability compared to the unstructured case. Observing the unstructured case the dark, densely packed region surrounding the trailing edge flaps suggests adaquate refinement. But, the problem with ICEM CFD is the lack of user control. The refinement seen around the flaps is unneccessary and doesn't refine the key features of the jet stream. 


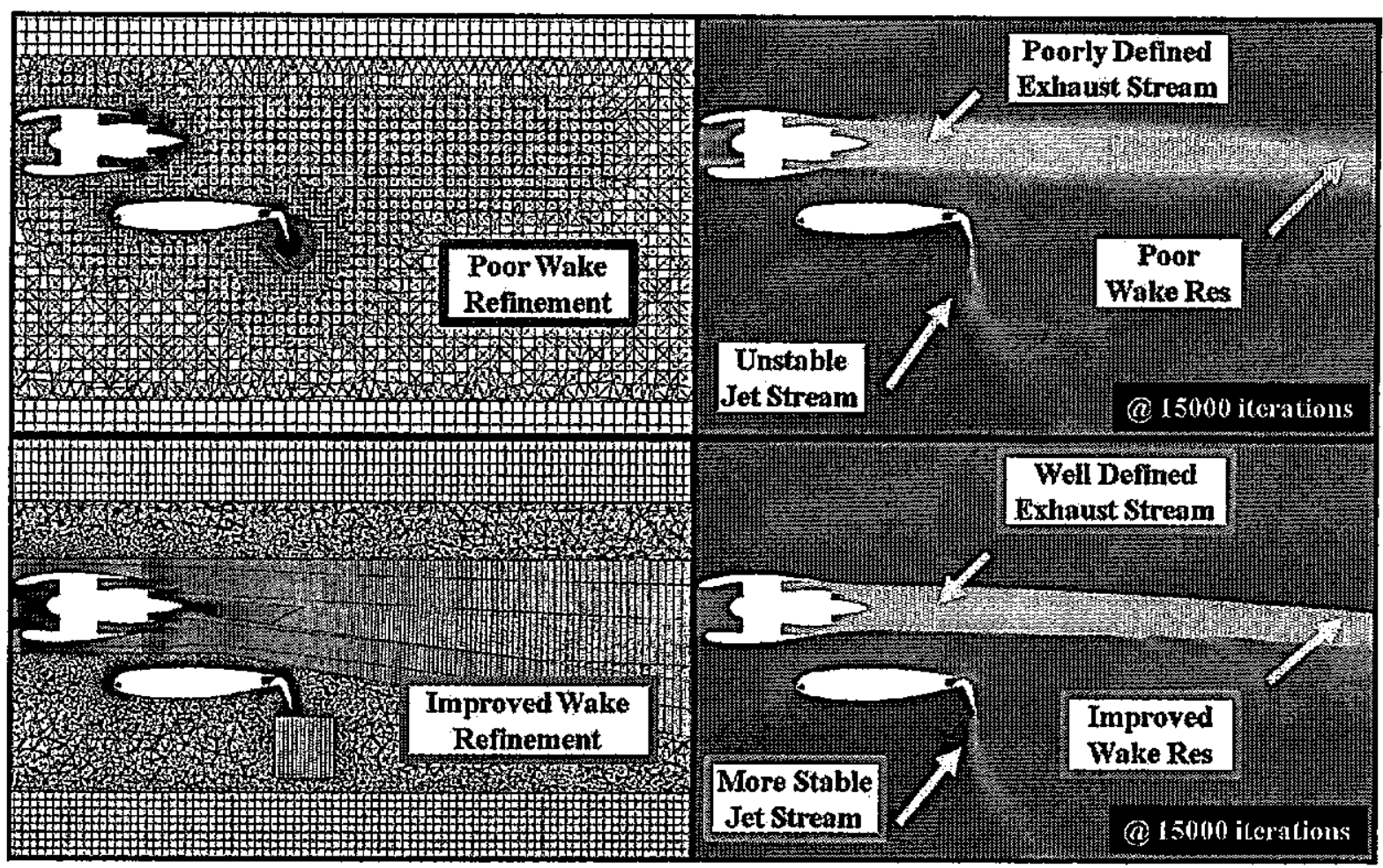

Figure 20. Comparison of CFD solution for different meshing techniques

Figure 21 shows a closer view of the engine exhaust. The first major problem we can see visually is the instability of the engine inlet for the unstructured case, that's not seen in the structured case. Despite both solutions simulated to 15,000 iterations, the engine inlet solution of the structured case appears to be well-behaved and has converged based on the boundary condition set at inlet face. The other concern is the jaggedness of the shear layer coming off the engine edges. Compared to the structured case, the solution is smeared and poorly defined. Futhermore, the jaggedness exhibited in the solution appears to resemble the shape of the unstructured elements. 


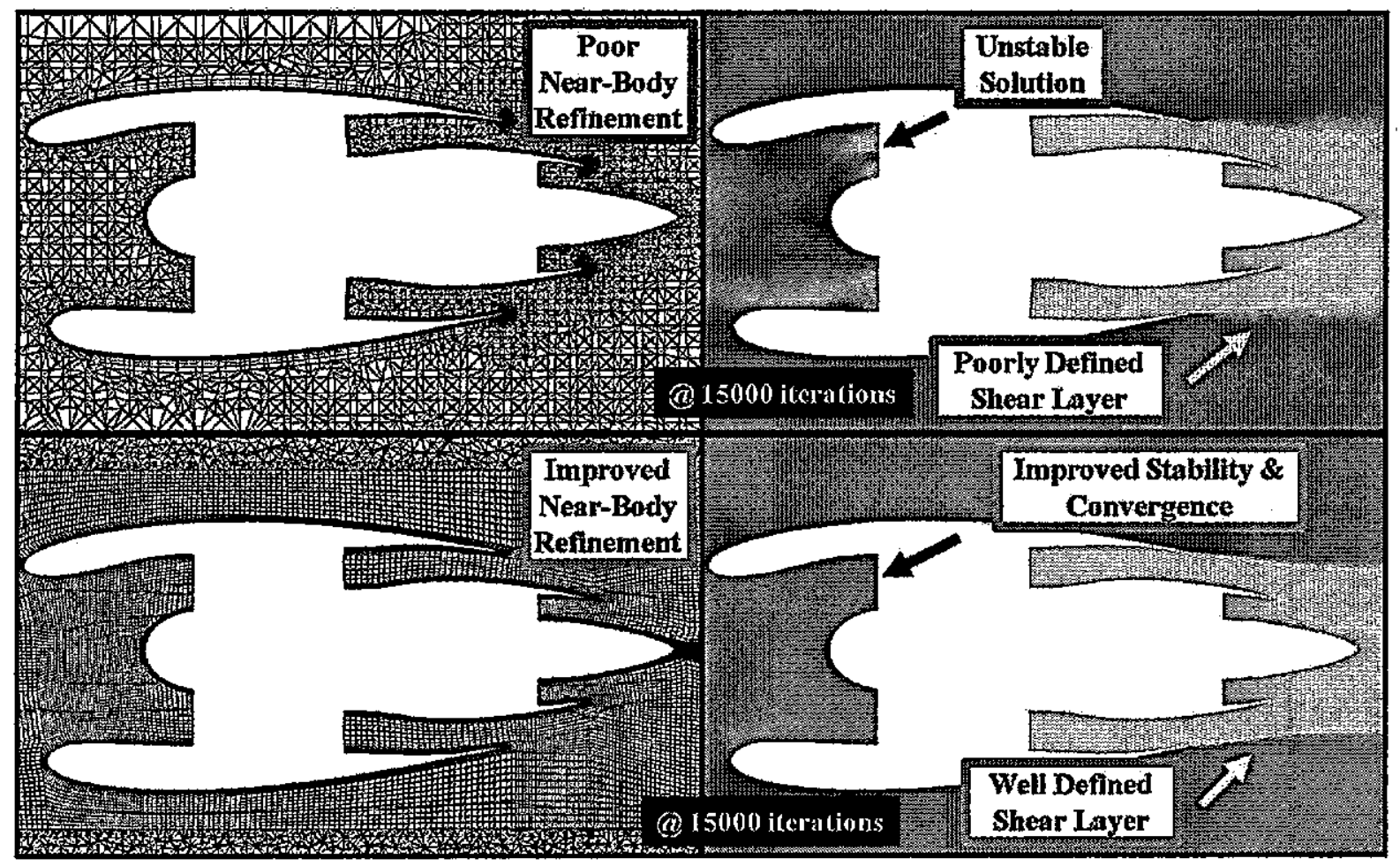

Figure 21. Comparison of CFD solution for different meshing techniques (close-view)

Figure 22 shows the final solution comparison. Observing the mesh, the structured case is much more refined cross-sectionally than the unstructured case. As seen in the figure, the mesh clearly plays a role in the poor annular resolution of the engine exhaust. In addition, the instability at the engine inlet can be seen more clearly in this figure. At this point in the simulation, there is reverse flow in the unstructured case as evident by the streamlines going upstream. When compared to the structured case, the streamlines are very well-behaved and doesn't appear to exhibit any flow issues. The behavior seen in the unstructured case has been observed before in preliminary analysis. Typically, the solution will eventually stabilize but this is going to require many more thousands of iterations. 


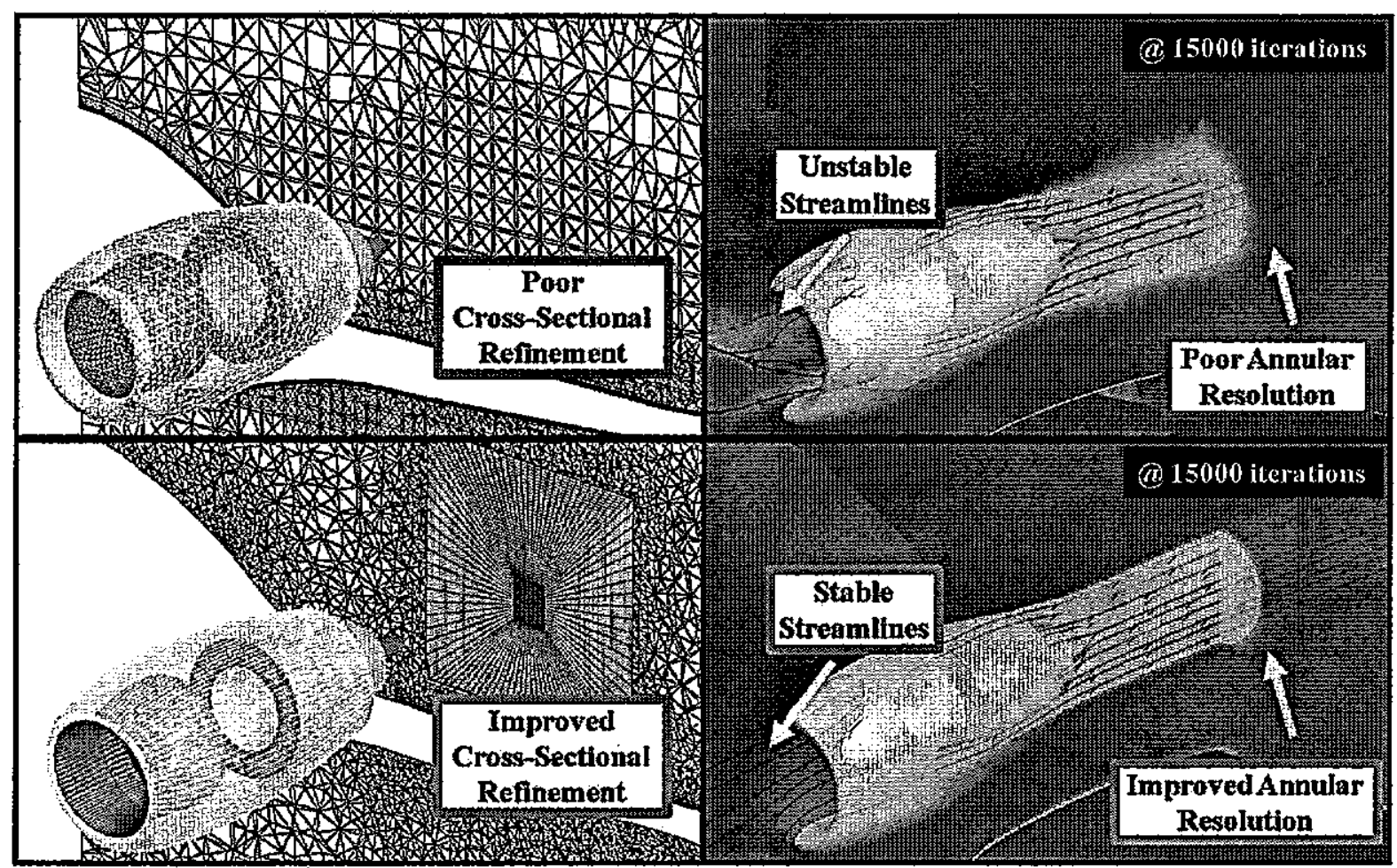

Figure 22. Comparison of CFD solution for different meshing techniques (isometric-view)

\section{Future Work}

There still exist many more concerns elsewhere with the computational model, especially around the leading and trailing edge circulation slots. Problems seen in this region are very similar to that of the engine exhaust, but it will be even more difficult to resolve this issue because the flow gradients in this region are almost twice as large and will require an even more resolved mesh. Similar to the engine mesh; the boundary layer, shear layer, and wake mesh will need to be resolved. In addition, the volume transition between the structured and unstructured elements will need to be smoothed out to prevent strange gaps in the CFD solution. These improvements are highlighted in Fig. 23.

Efforts toward improving the mesh quality have begun for the trailing edge circulation control flow region. This region includes the aft circulation control plenum and flaps. With more time, this blocking scheme will be extended to cover the entire wing. The multi-blocking scheme, surface mesh, and cutplane view of the volume mesh can be seen in Fig. 24 . 


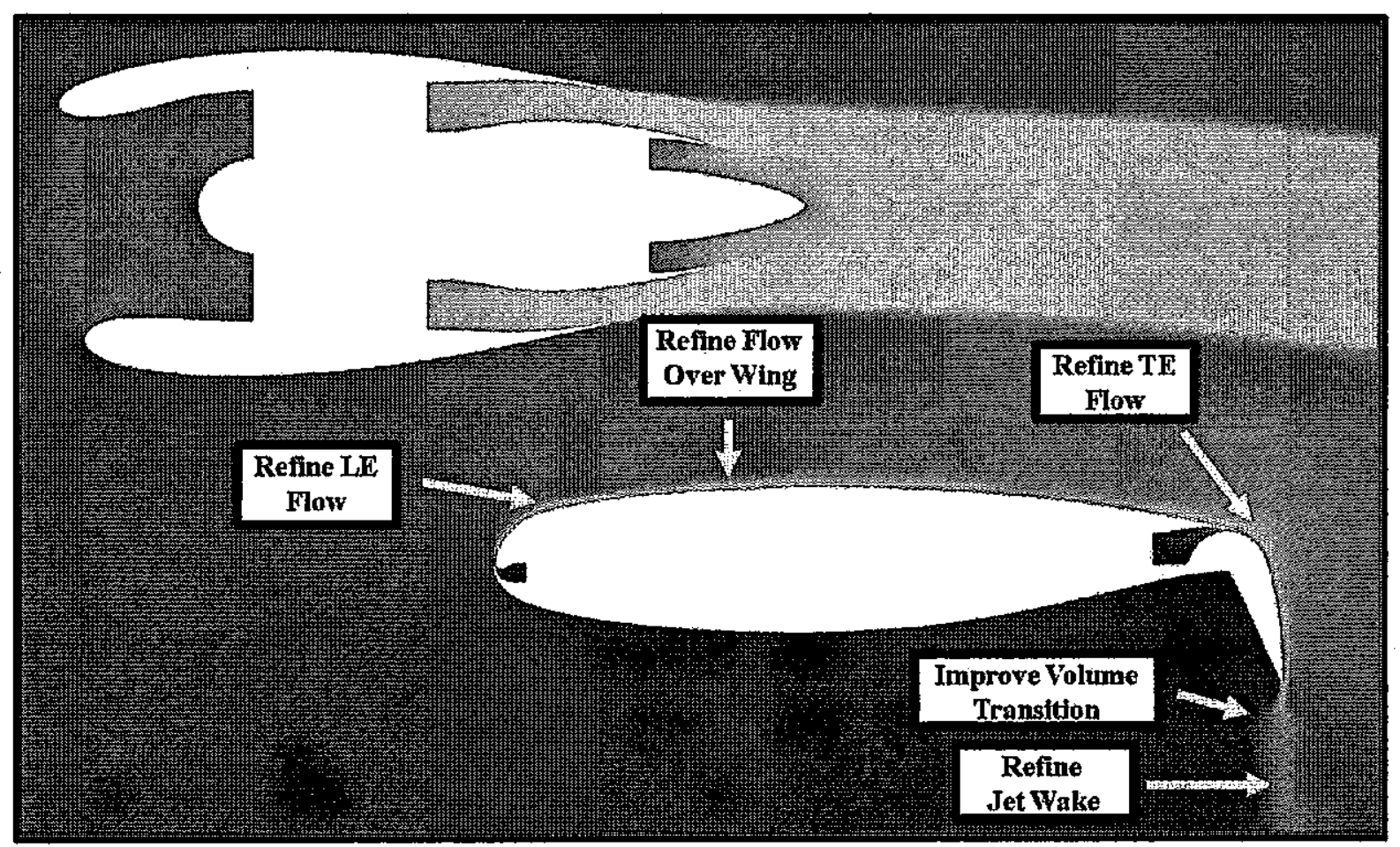

Figure 23, Regions that will require improvements in mesh quality
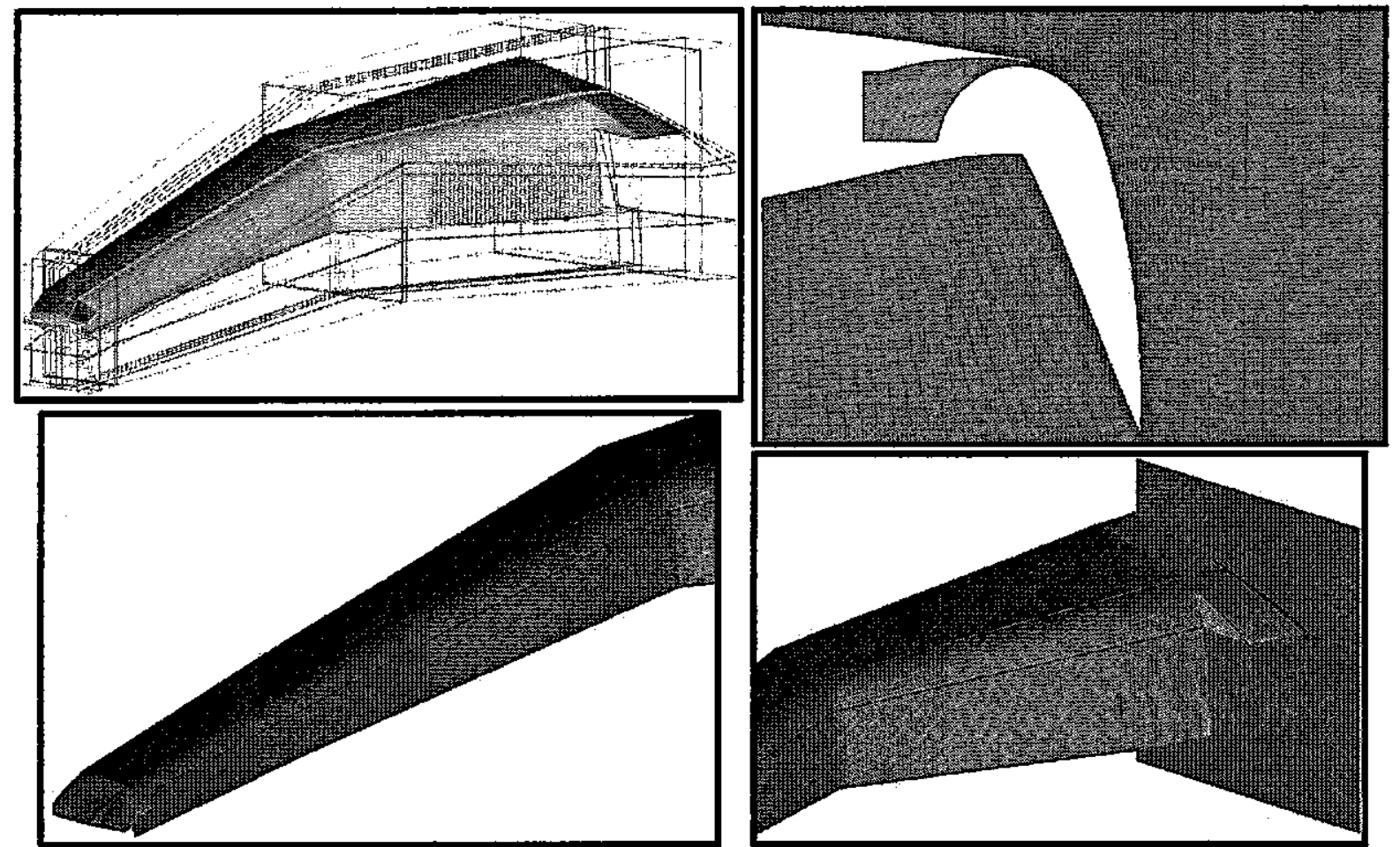

Figure 24. Multi-block scheme for trailing edge circulation control flow region 


\section{Concluding Remarks}

Unstructured CFD analysis for the AMELIA aircraft has yielded encouraging results and served as the basis for further improvement discussed in this paper. Numerical simulation of 3-D circulation control flow fields is a very difficult task as we continue to gain more knowledge of what techniques are best for predicting reasonably accurate CFD solutions. Concerns surrounding the 1st Method's ability to resolve the engine exhaust flow have been resolved using the 2nd Method. The 2nd Method has improved the quality of the CFD solutions immensely and will continue to be explored. Future work includes applying the techniques learned through the 2nd Method for the leading and trailing edge circulation control slots. Overall, there three concluding remarks.

1. The current computing resources at Cal Poly limit the total size of the mesh to about 35 million cells, thus constraining further refinement.

2. The current meshing software used, ICEM CFD, does not allow enough user control to refine particular regions in the flow field. Alternative codes will need to be explored in order for Unstructured Meshing to be sufficient for this NRA Project.

3. Given the total mesh size constraint, Structured Meshing clearly attains more refined and stable CFD solutions. Although time-consuming, this may prove to be the best method for predicting the complex flow physics of AMELIA with existing computing constraints.

\section{Acknowledgments}

This work was funded as part of a NASA Research Announcement award under Contract \#NNL07AA55C with Craig Hange and Joe Posey as the technical monitors. The authors wish to thank Rory Golden and Eric Paciano for their work in developing the computational model for meshing and numerical simulation. In addition, the authors would like to thank Bryan Blessing, Jay Marcos, and Jon Lichtwardt for their CFD contributions to the project.

\section{References}

\footnotetext{
1Englar, R., "Development of Circulation Control Technology for Powored-Lift STOL Aircraft," NASA Ames Research Center Proceedings of the Circulation-Control Workshop, 1987.

${ }^{2}$ Marshall, D. and Jameson, K., "Overview of Recent Circulation Control Modeling Activities at Cal Poly" AIAA-80100948, 48th AIAA Aerospace Sciences Meeting, 2010.

${ }^{3}$ Englar, R. and Huson, G., "Development of Advanced Circulation Control Wing High-Lift Airfoils" Journal of Aircraft, Vol. 21, No. 7, 1983.

${ }^{4}$ Panitz, T. and Wasan, D. T., "Flow Attachment to Solid Surfaces: the Coanda Effect," AYChE Journal, Vol. 18, 1972.

${ }^{5}$ Blessing, B., "Analyzing The Engine Entrainment of An Over the Wing Engine Coupled With A Circulation Control Wing," International Powered Lift, Philadelphia $P A, 2010$.

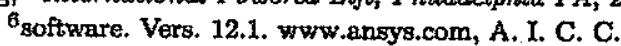

${ }^{7}$ Blessing, B. and Pham, J.s "Using CFD as a Design Tool on New Innovative Airliner Configurations," AIAA-2009-0045, 47th AIAA Aerospace Sciences Meeting, 2009.

${ }^{8}$ Marcos, J. and Marshall, D., "Computational and Experimental Comparison of Powered Lift, Upper Surface Blowing Configuration," AIAA-2010-0502, 48th AIAA Aerospace Sciences Meeting, 2010.

${ }^{9}$ Marcos, J. and Marshall, D., "Improved Computational and Experimental Valtdation Using Different Turbulence Models," AIAA-2010-4987, 28th AIAA Applied Aerodymamics Conference, 2010.

${ }^{10}$ Lichtward仑, J. and Marshall, D., "Investigation of Unsteady Behavior of a Circulation Control Wing Using Computational Fluid Dynamics," Submitted to 49th AlAA Aerospace Sciences Meeting, 2011.

${ }^{11}$ Lane, K. A. and Marshall, D. D., "Inverse Airfoil Destgn Utilizing CST Parameterization," AIAA-2010-1288, 48th AJAA Aerospace Sciences Meeting, 2010.

${ }_{12}$ Golden, R. and Marshall, D. D., "Dexign and Performance of Circulation Control Flap Systems," AIAA-2010-1059, 48th AIAA Aerospace Sciences Meeting, 2010.

${ }^{13}$ Storm, T. and Marshall, D. D., "Assessing the v2-f Turbulence Models for Circulation Control Applications," AIAA2010-1054, 48th AIAA Aerospace Sciences Meeting, 2010.

14 White, F., Visconts fluid fow, McGraw-Hill Higher Education, 2006.

${ }^{1 B}$ software. Vers, 6.3.26. www.ansys.com, A. F. C.

${ }^{16}$ Marshall, D., "The Integrated Modeling and Verification of Hybrid Wing-Body Low Noise FSTOL Atrcraft," NRA Contract No. NNLO7AA55C, 2010.
} 